\title{
The impact of teaching computational astronomy on the development of students' computer skills
}

\author{
Afrodita L. Boldea ${ }^{12 .}$ \\ 'Horia Hulubei National Institute for R\&D in Physics and Nuclear Engineering (IFIN-HH), \\ Bucharest \\ -Magurele, Romania \\ ${ }^{2}$ University of Craiova, Romania
}

\begin{abstract}
Some blended methods of teaching-learning were successfully used in teaching the astronomy and astrometry of asteroids to students in Computer Sciences at the University of Craiova, using real astronomical data about celestial objects from our Solar System, obtained from the Astronomical Observatory Isaac Newton (La Palma, Spain). The students were asked to develop some small scripts in order to facilitate the detection and the analysis of data for new discovered asteroids, a request that improved their capacities to understand and apply various modern concepts of Computer Graphics, Data Base and Web design. This approach to learning brings new challenges for the students, new opportunities for the process of professional training in Computer Sciences and provided good result in very short term, the students acquiring very fast the necessary skills to approach both the professional level of Web programming and the modern research area of Computational Astronomy.
\end{abstract}

\section{Introduction}

Astronomy today is undoubtedly one of the sciences which has exceptional public interest, promoted and boosted by the popular science broadcasts and the latest discoveries in the field. It can be said that Astronomy has influenced the perception of humans and their environment for millennia and is an excellent example of the interaction between science, technology and culture, in all its historical and contemporary issues. Astronomy is a science in itself, with its own methods (remote observations, photometry, spectroscopy), but also uses other scientific fields as instruments for understanding and modeling the Universe.

* Corresponding author: alinusha b@yahoo.com 
Teaching Astronomy can contribute to the understanding of physical laws, both on the human scale and cosmic scale, but it also offers a wealth of pedagogical examples of what the scientific approach means.

The integration of Astronomy in the teaching-learning process of Computer Sciences presents various advantages for the instruction process, first by improving the attitude of the students toward learning, second by the informational challenges that modern Astronomy can produces.

This paper presents the results of an interdisciplinary experimental methodicalscientific approach to the learning of Web technologies using the challenges in the field of Astronomy.

\section{Teaching Astronomy through Computer Sciences in the same class}

From 1995, the European Union has operated the EURONEAR project (European Near Earth Asteroid Research [1]), which deals with the identification of NEA (Near Earth Objects) and PHO (Potential Hazardous Objects). At present there are 15000 known NEA asteroids [2]; more that 1730 of them are PHOs.

In combination with this project, at University of Craiova was proposed an learning experimental approach to astronomy teaching, based on a blended-learning method that combines the classical teaching of astronomical concepts about the solar system with the application of the students' knowledge in the numerical processing of real photos of the sky, taken from a telescope from La Palma, and the development of some scripts that allow the identification of asteroids, their classification, or the determination of their orbital parameters. This paper describes the approach and results of the second year of this methodical experiment, while the results of the first year of the experiment were describe in [3].

The taught modules were given to a small number of students in Computer Science at the end of their second year of study, the activities being carried out partly at University of Craiova (in May 2017) and partly at "Horia Hulubei" National Institute for R\&D in Physics and Nuclear Engineering (IFIN-HH) in Magurele (June 2017), during a three-week internship in a professional practice.

The introduction of Computational Astronomy modules has been carried out in several stages, schematically depicted in Figure 1.

The Astronomy concepts familiarization stage (1) was conducted using classical two teaching methods, the stages of application of the theory in practice - the detection of asteroids in packets of images (2), and the determination of their coordinates and the calculus of the orbit (3) - use some specialized software applications: Astrometrica [4] and FindOrb [5] (see also Figure 2 


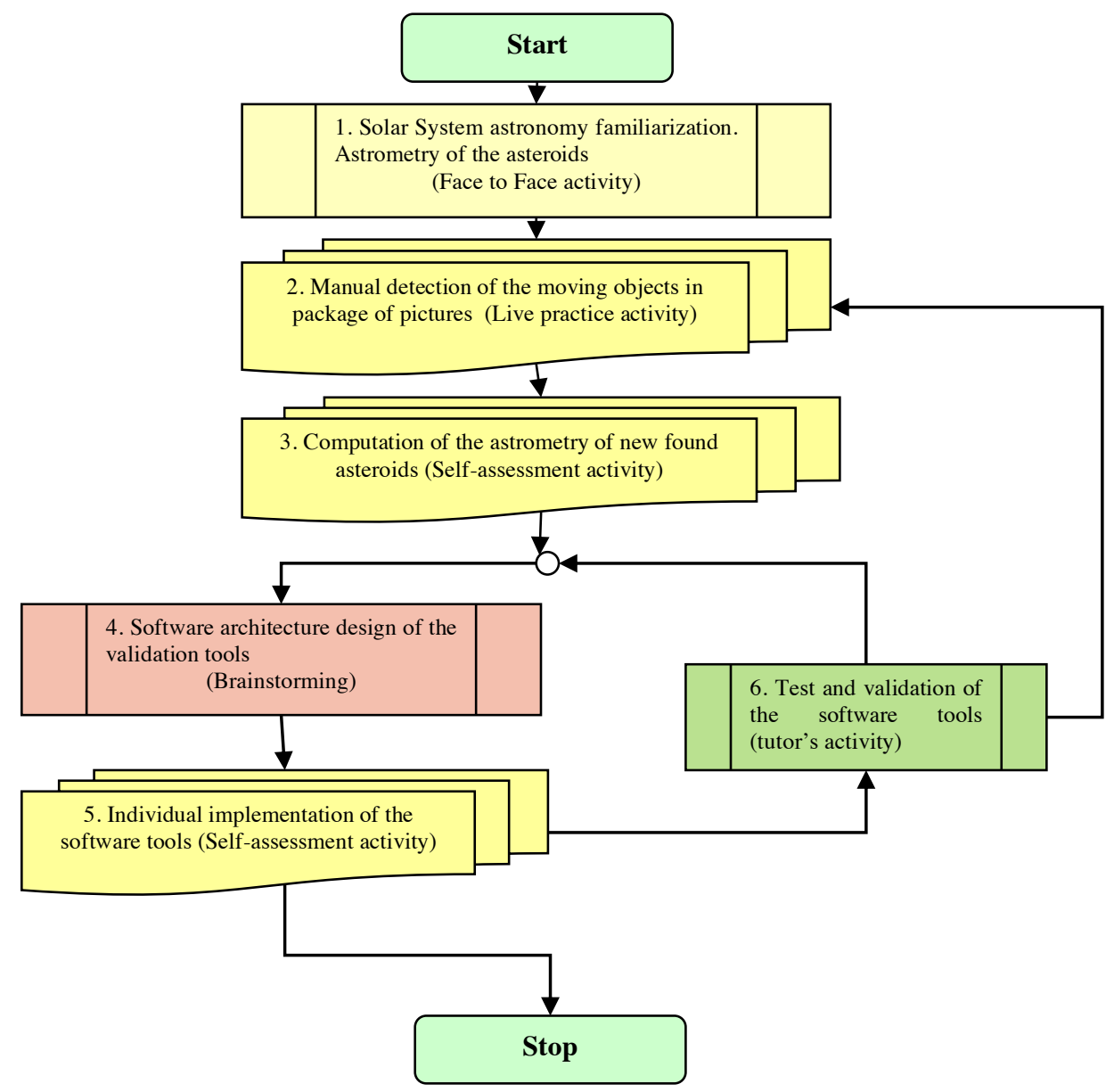

Fig. 1. The diagram of the computational astronomy learning modules
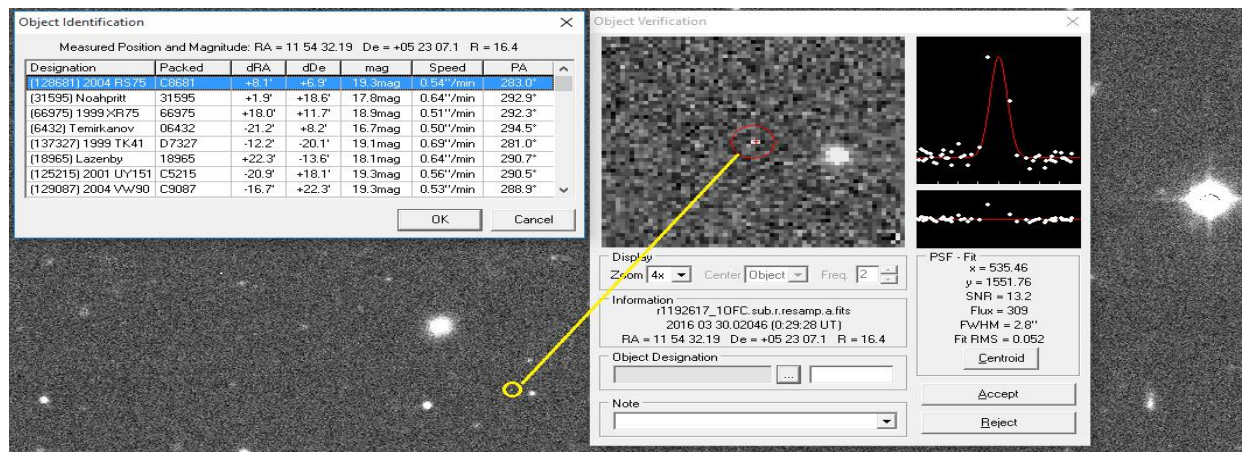

Fig.2 Detection of an asteroid using Astrometrica

The following table exemplifies the type of information obtained from the detection of an allegedly unknown asteroid (R.Asc. - Right Ascension, Decl. - Declination, Ap.Magn. Apparent Magnitude, Code obs. - the identification code of the Astronomical Observatory) 
Table 1. The centralized identification data in format MPC (Minor Planet Center) for a new asteroids candidates

\begin{tabular}{||cccccc||}
\hline $\begin{array}{c}\text { Temporary } \\
\text { name }\end{array}$ & $\begin{array}{c}\text { Date } \\
\text { (Julian format) }\end{array}$ & R.Asc & Decl. & $\begin{array}{c}\text { Ap. } \\
\text { Magn. }\end{array}$ & $\begin{array}{c}\text { Code } \\
\text { obs. }\end{array}$ \\
EURV071 & C2015 1103.9421823 & 3201.87 & +163952.3 & $21.2 \mathrm{R}$ & 950 \\
EURV071 & $\mathrm{C} 20151103.9432123$ & 3201.85 & +163951.9 & $21.4 \mathrm{R}$ & 950 \\
EURV071 & $\mathrm{C} 20151103.9442423$ & 3201.83 & +163951.6 & $21.1 \mathrm{R}$ & 950 \\
EURV071 & $\mathrm{C} 20151103.9452523$ & 3201.82 & +163951.2 & $21.2 \mathrm{R}$ & 950 \\
EURV071 & $\mathrm{C} 20151103.9462823$ & 3201.78 & +163950.9 & $21.3 \mathrm{R}$ & 950 \\
EURV071 & $\mathrm{C} 20151103.9473023$ & 3201.78 & +163950.7 & $21.4 \mathrm{R}$ & 950 \\
\hline
\end{tabular}

Next, during a three-week internship at IFIN-HH, students were asked to design and implement a number of PHP scripts that allow a user not necessarily expert to validate the accuracy of the results obtained with Astrometrica, to determine whether a previously detected moving object is a known asteroid or not, to classify newly discovered objects (from main belt Asteroids, NEA or PHO), or to investigate which of the asteroids recorded in an international database should have appeared in the studied images.

Their elaboration required the acquisition of solid programming knowledge in PHP, Web Design and the use of more popular astronomical databases. The student's script design stage (4) was conducted under the direct guidance of the tutor (author of this article) in the form of a brainstorming, identifying, for each script, the way to enter the input data, how to access a database containing information about known asteroids, the types of numerical processing to be implemented, and the type of graphics used for displaying the results.

In the final stages (5-6), the students have individually implemented the scripts, using their knowledge from the two basic courses of the 2nd year (Web Technologies and Foundations of Databases), as well as important Web resources for completing this knowledge to the level required for elaboration of required scripts. We present below two of the software tools produced by the students in collaboration with the author.

\section{The students' software tools}

\section{a. The determination of the variation of the asteroid's orbit}

The Observed minus Calculated $(\mathrm{O}-\mathrm{C})$ Computer script determines the difference between calculated and observed positions of a known asteroid for a night of observations, as defined by the EURONEAR site [1].

The O-C Computer script uploads a set of astronomical observations (in MPC format), determines the name of the asteroid from the NeoDyS-2 database [6] that corresponds to the astronomical observations, interrogates the same database to determine the ephemeris (asteroid positions calculated according to identification data in the database) corresponding to the date and time of the observation file, with precision of a second, then calculates and graphically displays the differences (in arc seconds) between the observed positions and those determined by the calculation of ephemeris in universal equatorial coordinates. 
The application allows for rapid identification of observations with errors too large to be taken into account (when the scattering of the residuals is greater than a few seconds of arc), or, alternatively, causes a slight orbital deviation of the asteroid (when the residuals are grouped in a region clearly separated from the origin), in which case it is necessary to compute a correction of the known asteroid trajectory.

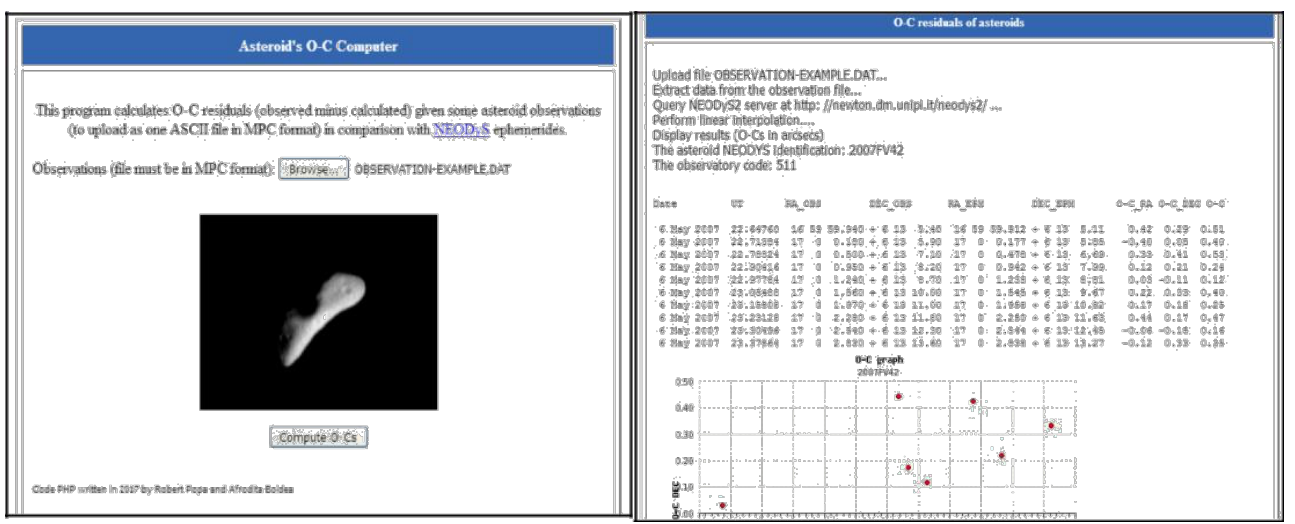

Fig. 3. The O-C Computer Script

\section{b. The evaluation of Earth impact risk by an asteroid (The Mu-Eps Scatter Plotter)}

The Mu-Epsilon Scatter Plotter program takes a set of astronomical observations (also in MPC format), determines the apparent motion of the object in the sky (the Proper Motion in arc seconds per minute), both horizontally (RA) and vertical (DEC), as well as its apparent global motion $(M u)$.

It also determines the Solar Elongation (Eps), which represents the angle made by the Sun, Earth and the asteroid at the time of observation. The ratio of the two sizes makes it possible to quickly identify asteroids that have a high orbit (less eccentric) radius below the 1.3 UA limit, asteroids that are by definition classified as Near Earth Objects (NEAs) and which may pose a risk of impact with Earth. The determination of the NEA limit was made on the basis of a formula proposed in [7].

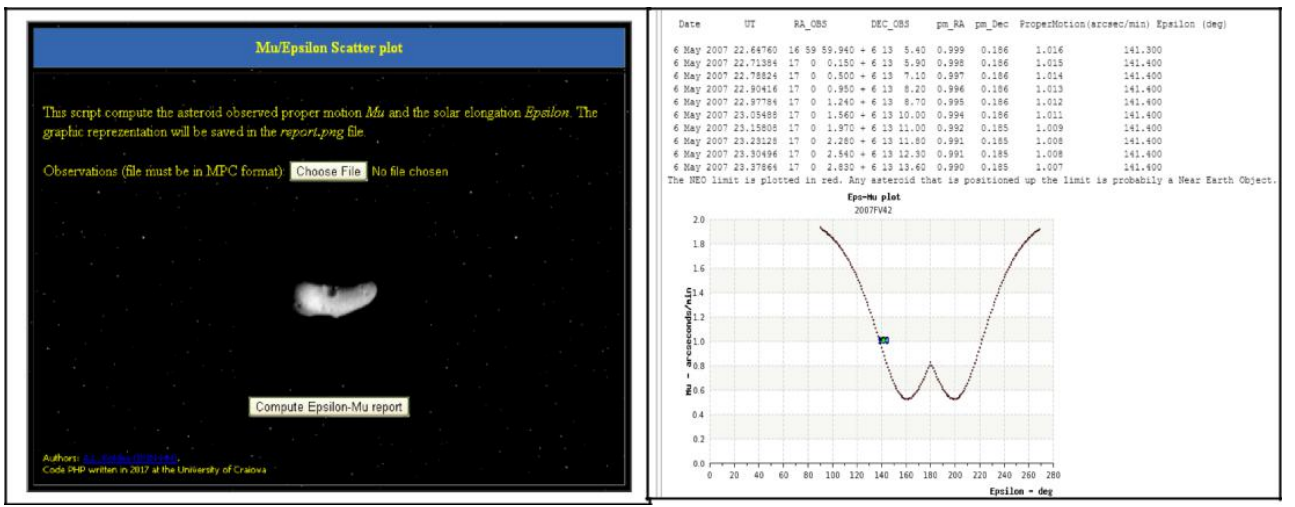

Fig. 4. The Mu-Eps Scatter Plotter 


\section{Conclusions}

Following this methodological experiment it was found that the students involved responded enthusiastically to the proposed themes, reaching within a short time (three weeks) the advanced practical skills of Web programmers, which was only expected at the end of the last year of university study in Computer Sciences.

In conclusion, Astronomy can serve as a launch pad for many areas of knowledge and human activities. Its multidisciplinary character is twice as exciting, given the possibility of showing students that knowledge is not as fragmented as it appears in study programs and also to prove that the evolution of knowledge takes place only by nature multidisciplinary approaches. For students in Informatics, the interest in astronomy is actually a powerful lever that allows them to illustrate, develop and validate a school education already received, but also to become interested in world scientific research.

\section{Acknowledgements}

This work was supported by the Ministry of Research and Innovation under project PN16420202.

\section{References}

[1] EURONEAR project, http://www.euronear.org/people.php (accessed in 2017)

[2] Minor Planet Center, http://www.minorplanetcenter.net/iau/mpc.html, (accessed in 2017)

[3] A L Boldea and O Văduvescu, "Teaching students about informatics and astronomy using real data for detection of asteroids", Eur. J. Phys. 38 (5), 055706 (2017)

[4] H. Raab, Astrometrica - Shareware for research grade CCD Astrometry, www.astrometrica.at/ (accessed in 2017)

[5] Project Pluto - On-line FindOrb, https://www.projectpluto.com/fo.htm, (accessed in 2017)

[6] NeoDyS-2, http://newton.dm.unipi.it/neodys/index.php?pc=0, accessed in 2017

[7] O. Văduvescu et al., "EURONEAR-Recovery, follow-up and discovery of NEA's and MBA's using large field 1-2m telescopes", Planetary and Space Science 59, 1632-1646 (2011) 